\title{
Breast conserving surgery versus modified radical mastectomy
}

Breast conserving therapy (BCT) or the so-called "lumpectomy" for breast cancer aims to achieve long-term local disease control with reduced local morbidity, improved quality of life, and many less operations if reconstructive surgery is advisable [1]. Evidences have accumulated to suggest that BCT has similar long-term survival outcomes to mastectomy in patients with early breast cancer. An increasing number of studies have shown improved overall survival among women treated with BCT regardless of cancer phenotype compared with mastectomy, particularly with the advent of increasingly effective neoadjuvant treatment [2]. It may no longer be appropriate to offer women suitable for BCT the choice of mastectomy or BCT.

The accuracy of patient stratification of early breast cancer is crucial for the success of BCT. Patients with clinical stage I, IIA, or a subset of stage IIB disease (T2N1) are classified as having early stage breast cancer [3]. In addition, the appropriate margin width for breast conservation surgery and radiation will need diagnostic strategies, which may not be readily available in many centers $[4,5]$. Therefore, it is conceivable that the accuracy of appropriate margin width for surgery and for radiation therapy will vary, particularly in centers where diagnostic tools are not sophisticated.

Vongsaisuwon et al. in this issue [6] reported a propensity score matched analysis of BCT versus mastectomy. They were able to confirm that the two treatment strategies for early breast cancer result in similar long-term local disease control, survival benefits and other cancer-specific outcomes. The analysis was performed on patients seeking care from an advanced university hospital with high standards of patient stratification, sentinel lymph node biopsy, and good estimates of margin with for surgery and radiation. In addition, the standards are also sophisticated for estimating tumor size, histologic grade, and the presence of lymphatic invasion within the primary tumor and selection of adjuvant treatments. Such standards might not be present in other less sophisticated centers. Thus, many surgeons in less sophisticated centers have frequently experienced recurrence after BCT and the patients eventually have ended up with radical mastectomy.

In addition, the preferences of patients frequently vary with age. Younger patients tend to opt for BCT, while older patients frequently prefer radical mastectomy. The main reasons for choosing mastectomy over BCT were fear of cancer recurrence, the perception that health outweighs breast retention and the possibility of second surgery for margins [7]. Key factors for rejecting immediate reconstruction after mastectomy were patient-perceived "old age", concern about two sites of surgery, and financial cost [7]. Given a second chance, many patients would undergo BCT instead of mastectomy [7].

Therefore, it cannot be overemphasized that sharing decisions with patients and relatives about the diagnostic and treatment options is of paramount importance. Presenting how the outcomes of diagnosis and treatments might vary depending on the contexts and varying capacities of healthcare system is an intrinsic art that providers in all settings have to master.

\section{References}

[1] Johns N, Dixon JM. Should patients with early breast cancer still be offered the choice of breast conserving surgery or mastectomy? Eur J Surg Oncol. 2016; 42:1636.

[2] Hyams DM, Schuur E, Angel Aristizabal J, Bargallo Rocha JE, Cabello C, Elizalde R, el al. Selecting postoperative adjuvant systemic therapy for early stage breast cancer: a critical assessment of commercially available gene expression assays. J Surg Oncol. 2017; 115:647-62. doi:10.1002/jso.24561.

*Correspondence to: Editorial Office of Asian Biomedicine, Faculty of Medicine, Chulalongkorn University, Bangkok 10330, Thailand, e-mail: abmjournal@chula.ac.th

O Open Access. ๑ 2019 Editorial Office of Asian Biomedicine, published by Sciendo. (๔) BY-NC-ND This work is licensed under the Creative Commons Attribution NonCommercial-NoDerivatives 4.0 License. 
[3] Russnes HG, Lingjærde OC, Børresen-Dale AL, Caldas C. Breast cancer molecular stratification: from intrinsic subtypes to integrative clusters. Am J Pathol. 2017; 187:2152-62. doi:10.1016/j. ajpath.2017.04.022.

[4] Moran MS, Schnitt SJ, Giuliano AE, Harris JR, Khan SA, Horton J, et al. Society of Surgical Oncology-American Society for Radiation Oncology consensus guideline on margins for breastconserving surgery with whole-breast irradiation in stages I and II invasive breast cancer. J Clin Oncol. 2014; 32:1507-15.

[5] He T, Puppala M, Ezeana CF, Huang YS, Chou PH, Yu X, et al. A deep learning-based decision support tool for precision risk assessment of breast cancer. JCO Clin Cancer Inform. 2019; 3:1-12. doi:10.1200/CCI.18.00121.

[6] Vongsaisuwon M, Pongpirul K, Chatanara K. Clinical outcomes and surgical preferences for breast-conserving surgery and mastectomy: a propensity score matched analysis. Asian Biomed (Res Rev News). 2019; 13:XX.

[7] Lee WQ, Tan VKM, Choo HMC, Ong J, Krishnapriya R, Khong S, et al. Factors influencing patient decision-making between simple mastectomy and surgical alternatives. BJS Open. 2018; 3:31-7. doi:10.1002/bjs5.50105. 